\title{
Supporting Information for \\ Computational Study of Magic-Size CdSe Clusters with Complementary Passivation by Carboxylic and Amine Ligands
}

Oleksandr Voznyy, Junais Habeeb Mokkath, Ankit Jain, Edward H. Sargent, Udo Schwingenschlogl
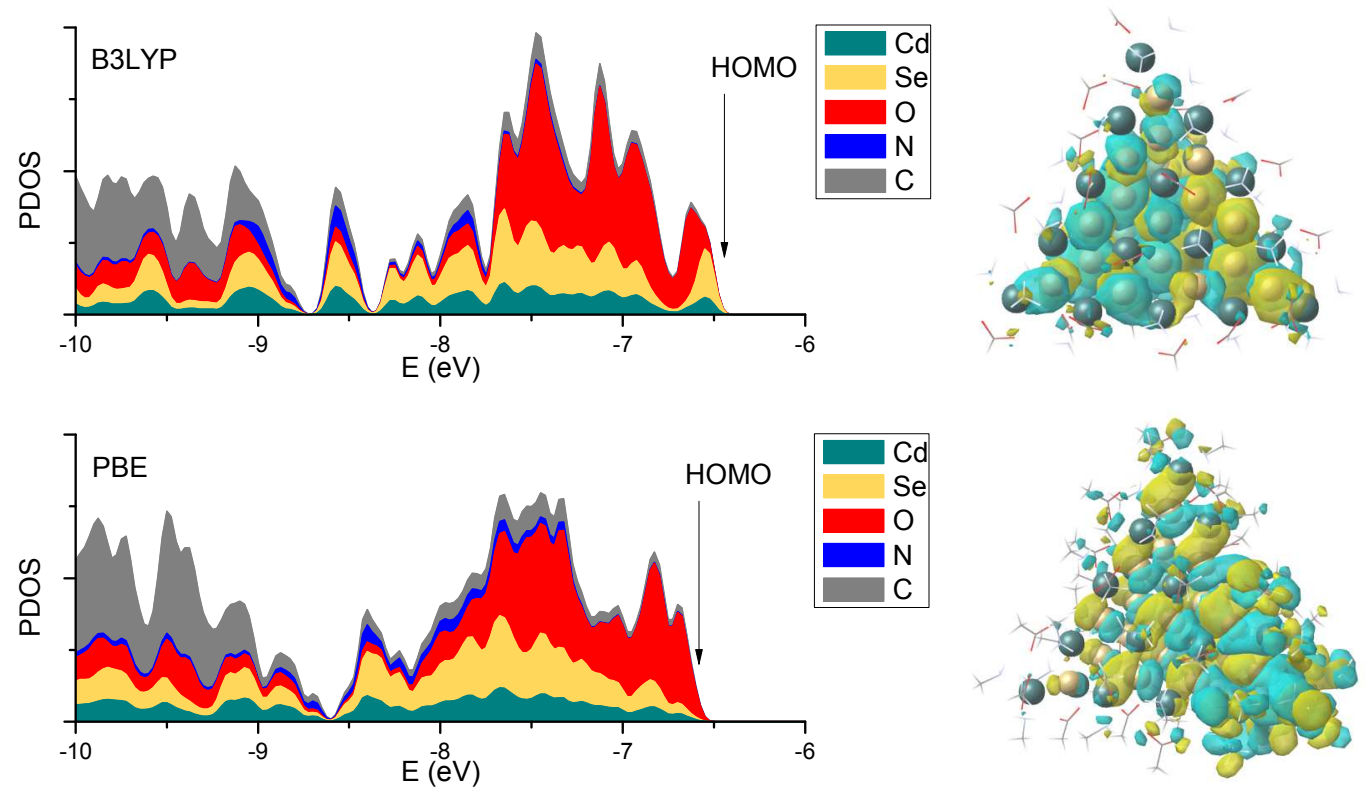

Figure S1. Calculated projected density of states and HOMO wavefunctions of the $\mathrm{Cd}_{35} \mathrm{Se}_{20}$ cluster with ammonium+acetate ligands.

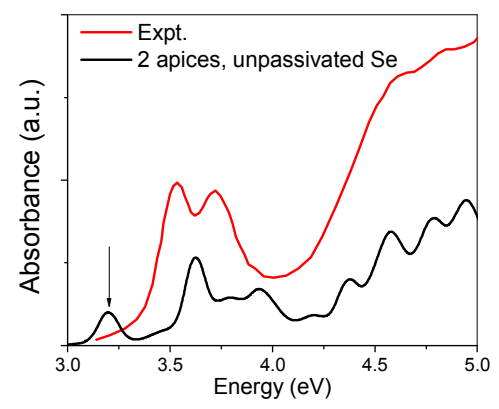

Figure S2. Calculated absorption spectrum of a cluster with 2 apices removed. Exposed Se dangling bonds are left unpassivated, while all $\mathrm{Cd}$ dangling bonds are passivated with pseudohydrogen, mimicking the experimental conditions. Exposed Se dangling bonds form trap-like states (arrow), resulting in additional sub-bandgap transitions. 